\title{
INVESTIGATING THE INFLUENCE OF THE CONSTITUENT MATERIALS ON THE PERFORMANCE OF PERIODIC PIEZOELECTRIC COMPOSITE ARRAYS
}

\author{
A Gachagan, G Harvey, RL O'Leary and J. Mackersie \\ Centre for Ultrasonic Engineering, Department of Electronic and Electrical Engineering, \\ University of Strathclyde, 204 George Street, Glasgow, G1 1XW, UK
}

\begin{abstract}
This paper describes a theoretical investigation into the influence of the constituent materials on periodic composite array transducer performance. A finite element (FE) model, configured in PZFlex, is used to analyze the performance of a wedge coupled array transducer operating into a steel component. Here, the improvements offered by new single crystal piezoelectric materials are compared to standard PZT-based configurations. In addition, new passive polymer materials, possessing low longitudinal loss and high shear loss, are evaluated for their potential to significantly reduce interelement mechanical cross talk. The FE results illustrate the potential for the next generation of array transducers incorporating these new materials and this is highlighted in the A-scan predictions from simulated defects.
\end{abstract}

Keywords: Finite Element Modeling; Phased Array Simulation; Imaging Performance PACS: 81.70Cv; 07.05.Tp

\section{INTRODUCTION}

Ultrasonic transducer arrays are regularly employed in both biomedicine [1] and sonar systems [2] and are now finding application in a number of inspection procedures within NDE [3]. The principal advantages of array technology over conventional single element probes include enhanced inspection speed, increased coverage and versatility from a single probe location, as one array probe can undertake many inspection scenarios. Although array technology has existed for a number of years, the principal drawback to its widespread use for NDE applications has been the availability and cost of the necessary array controller instrumentation. This is no longer an issue, however, with several commercial phased array controllers available on the market.

In most modern ultrasonic array configurations, the piezoelectric ceramic composite structure, comprising an array of piezoceramic elements embedded within a polymer matrix, is commonly used as the active material. Since the mid 1980's, there has been significant research activity attributed to the design of the active and passive phases within a piezoelectric composite configuration [4-7]. Importantly, the selection of the constituent materials is application specific and crucial to the success of the design process. Although, this technology is fairly mature, new advances in piezoelectric and polymeric materials have been reported in recent years and have been shown to improve array sensitivity, bandwidth and imaging performance in biomedical [8] and sonar [9] applications. This paper aims to determine if these same potential benefits can be realized when these new materials are incorporated into an NDE array configuration. 
This paper describes the application of finite element (FE) modeling techniques to investigate the influence of the constituent materials on periodic composite array transducer performance. The NDE scenario under consideration is a wedge coupled array transducer operating into a steel component containing a side drilled hole (SDH) defect. The improvements offered by new single crystal piezoelectric materials are compared to standard PZT-based configurations and in addition, new passive polymer materials, possessing low longitudinal loss and high shear loss, are evaluated for their potential to significantly reduce inter-element mechanical cross talk. The FE results demonstrate a significant improvement in array bandwidth for piezoelectric composite array transducers incorporating these new active and passive materials.

\section{THEORY}

\section{Piezoelectric Ceramic Composite Structure}

The typical piezoelectric composite substrates utilized in ultrasonic arrays are termed either 2-2 connectivity or 1-3 connectivity configurations [4]. Figure 1 illustrates these two configurations in which the first number refers to the connectivity of the active piezoelectric phase and the second to the connectivity of the passive polymer phase. One critical design parameter is the pillar aspect (width-to-height) ratio in the 1-3 configuration, which must be minimized to ensure efficient transducer operation [6]. Moreover, tailored selection of the constituent materials will define the transducer performance in terms of sensitivity, bandwidth and imaging performance.

Recently, a number of piezoelectric single crystal materials have been reported as offering significant benefits over conventional piezoceramic materials in terms of sensitivity and bandwidth [8,9]. In order to take advantage of these potential benefits, the single crystal material has to be correctly configured in a piezoelectric composite structure. For this paper, the Perovskite single crystal material $\mathrm{Pb}(\mathrm{Mg} 1 / 3 \mathrm{Nb} 2 / 3) \mathrm{O}_{3}-\mathrm{PbTiO}_{3}(\mathrm{PMN}-$ PT) is used as the active phase [9].

A new polymeric material has been developed at Strathclyde for implementation in piezocomposite array configurations [10]. This material exhibits anisotropic elastic properties and importantly, high shear loss and low longitudinal loss characteristics, as detailed in Table 1, to minimize mechanical cross-coupling across the transducer array structure. Consequently, this 'hybrid' polymer material inhibits the generation of Lamb waves in these periodic structures, which have been shown to be responsible for degrading array imaging performance [11].

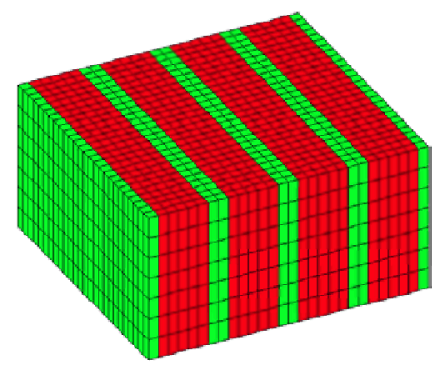

(a) 2-2 Connectivity

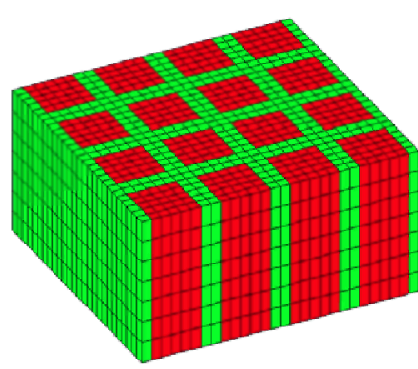

(b) 1-3 Connectivity

FIGURE 1. Piezoelectric ceramic composite configurations utilized in ultrasonic array transducers. 
TABLE 1. Material properties of the polymeric materials used in this study.

\begin{tabular}{c|c|c|c|c} 
Material & $\begin{array}{c}\text { Longitudinal } \\
\text { Velocity }\end{array}$ & Shear Velocity & $\begin{array}{c}\text { Longitudinal } \\
\text { Attenuation }\end{array}$ & $\begin{array}{c}\text { Shear } \\
\text { Attenuation }\end{array}$ \\
\hline \hline Hard Polymer & 2513 & 1175 & 139 & 356 \\
\hline Hybrid Polymer & 1179 & 602 & 143 & 9554
\end{tabular}

\section{NDE Inspection Scenario under Consideration}

A 32-element linear array is bonded onto a Rexolite wedge and designed to operate at $1 \mathrm{MHz}$. The array electrode pattern is defined by the half wavelength spacing. The wedge is used to couple ultrasonic energy into a steel block and a focal law has been applied to produce a pressure focus $30 \mathrm{~mm}$ from the surface, at an angle of $45^{\circ}$. This inspection scenario is illustrated in Figure 2, with a SDH located at the focal point and indicated by the white circle in the steel component. This defect will be used to illustrate the respective imaging performance of different piezoelectric composite array configurations.

\section{Finite Element Modeling}

The finite element code, PZFlex [12], has been used to simulate four piezoelectric composite configurations combining PZT5H and PMN-PT as the active piezoelectric phase and the hard and hybrid polymer materials (see Table 1) as the passive phase. Importantly, a 2D model has been employed to minimize the computational requirements and therefore, this model can be considered representative of both 1-3 and 2-2 connectivity composite configurations. Due to the different longitudinal velocities for both the passive and active phases used in this study, each transducer design will be slightly different in terms of transducer thickness and pillar aspect ratio. Significantly, each device is designed to operate at $1 \mathrm{MHz}$ (at electrical resonance) and exhibit uni-modal characteristics to ensure that the aspect ratio criterion is fulfilled [6]. This is illustrated in the electrical impedance characteristic shown in Figure 3, for a PZT5H/Hard Polymer combination with a monolithic electrode applied to the top and bottom faces. It should be noted that the impedance characteristic across each of the four configurations was consistent in this respect.

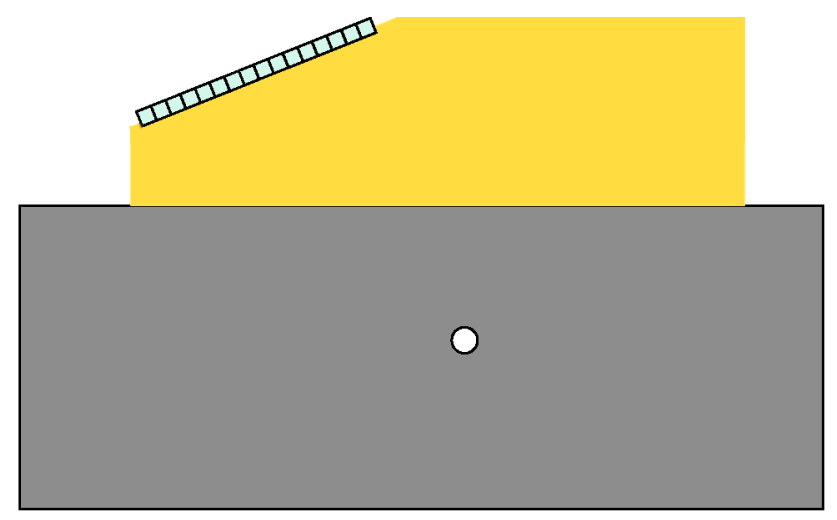

FIGURE 2. Illustration of NDE scenario used in the evaluation process. 


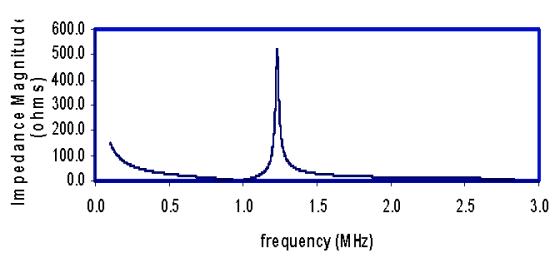

(a) Magnitude

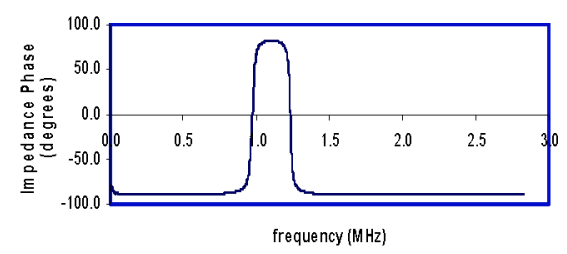

(b) Phase

FIGURE 3. Electrical impedance characteristic illustrating uni-modal behavior.

\section{RESULTS}

\section{Characterization of the Array Configurations}

It is necessary to understand that when a composite structure is configured with a linear array electrode pattern, the electrical impedance characteristic will be modified due to the presence of Lamb waves. This is a consequence of the periodic nature of the piezoelectric substrate and array pattern [11]. This is clearly illustrated in Figure 4, which presents the electrical impedance characteristic for a single array element in the PZT5H/Hard polymer configuration. When compared to the corresponding magnitude and phase response shown in Figure 3 for a monolithic electrode arrangement, the corruption of the uni-modal characteristic is evident in the phase response, in particular. Importantly, it must be noted that for the two configurations utilizing the new 'hybrid' polymer material there was no difference in the electrical impedance characteristic between the monolithic and array electrode configurations.

Next, FE modeling was used to predict the mechanical cross-coupling across each array aperture to assess the influence of the new 'hybrid' polymer to inhibit the generation of parasitic Lamb wave activity. The 'in air' surface displacement characteristics predicted for the PZT5H/hard and PZT5H/hybrid composite configurations are shown in Figures 5 (a) and 5(b) respectively. Here, a half symmetry model has been utilized and hence, the two centre elements are electrically excited, evident in both Figures, where the predicted maximum displacement values are similar at around $2.5 \mathrm{~nm}$. The interesting phenomenon is the surface displacement in the adjacent electrodes and across the entire array aperture. The superior shear damping associated with the 'hybrid' polymer has produced very low levels of displacement across the array structure (Figure 5(b)). It is this array behavior that has been reported to improve the device imaging performance without compromising element sensitivity [10].

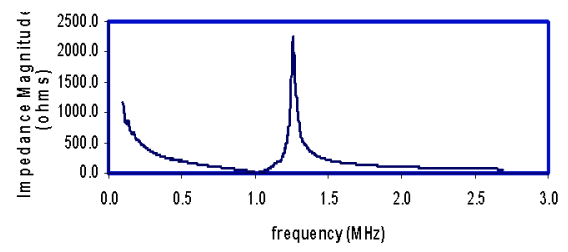

(a) Magnitude

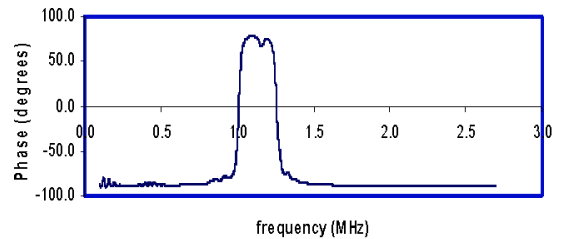

(b) Phase

FIGURE 4. Electrical impedance characteristic for a single element in the 2-element array configuration. 


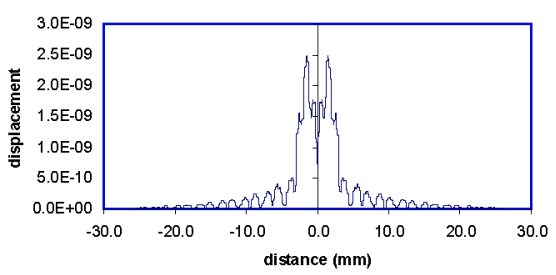

(a) PZT5H/hard composite

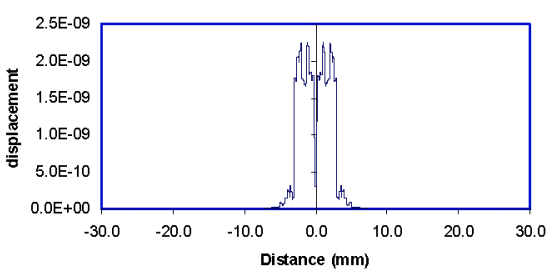

(b) $\mathrm{PZT} 5 \mathrm{H} /$ hybrid composite

FIGURE 5. Predicted surface displacement characteristic

\section{$\underline{\text { Predicted Peak Pressure Field Profile }}$}

The conventional method for depicting the pressure field from a phased array transducer is to map peak pressure. This will highlight the principal focal region for the transducer configuration and focal law combination, in addition to any spurious ultrasonic energy from sidelobes due to incorrect electrode spacing. Hence, this approach was used to assess the pressure field characteristic of each transducer configuration and Figures 6(a) and 6(b) present the predicted field response for the two transducer configurations using PZT5H as the active material. Interestingly, there is very little difference between these fields, which indicates that the performance of transducers incorporating either the hard or 'hybrid' polymer material as the passive phase is similar. On further consideration, the 'hybrid' polymer phase is designed to inhibit mechanical cross-talk between elements in the array configuration, which will be of a lower amplitude than the initial burst of ultrasonic energy. Hence, this secondary piezoelectric generation will not be represented in this peak pressure field prediction and alternative methods are required to quantify the advantages associated with the new 'hybrid' polymer.

\section{Predicted Propagating Pressure Field Profile}

The next stage was to analyze the wave generation and propagation with time using the FE models. This enables the initial burst of energy and the secondary generation due to mechanical cross-talk to be visualized simultaneously. Two examples of this are presented in Figures 7(a) and 7(b) for the two most extreme cases: PZT5H/hard composite and PMNPT/hybrid composite. This snapshot in time corresponds to the pressure field after passing through the desired focal point and the main wave packet (approximately 4 cycles) is clearly evident in the bottom right hand corner of each Figure. The notable feature is the difference in the ringing after this main wave packet. This is due to the narrower bandwidth associated with the conventional PZT materials when compared to the new single crystal materials and the reduced mechanical cross-talk associated with the composite design incorporating the 'hybrid' material.

\section{Reconstructed Pulse-Echo Array Response}

The final stage in this evaluation process was the inclusion of a $5 \mathrm{~mm}$ radius $\mathrm{SDH}$ defect in the model and subsequent, reconstruction of the pulse-echo response from each composite array configuration using the focal law specification. Figures $8(a)$ and $8(b)$ show $\mathrm{B}$-scan data corresponding to the pulse-echo response from each array element. The diagonal wavefront is attributable to the echo from the wedge/steel interface, with the signal at approximately $35 \mu$ s corresponding to the echo from the defect. Significantly, 


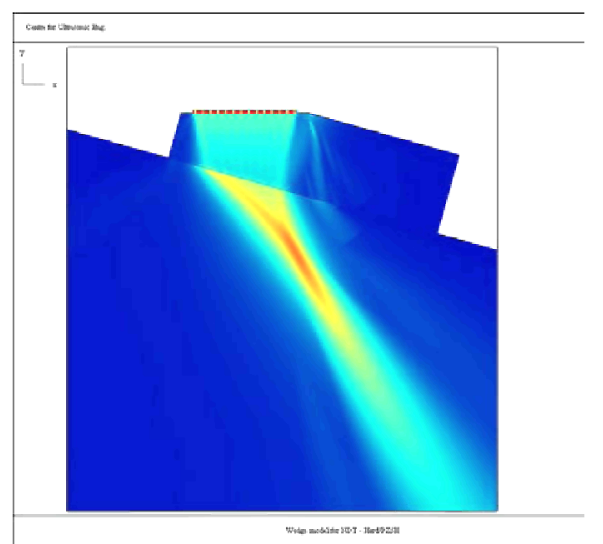

(a) PZT5H/hard configuration

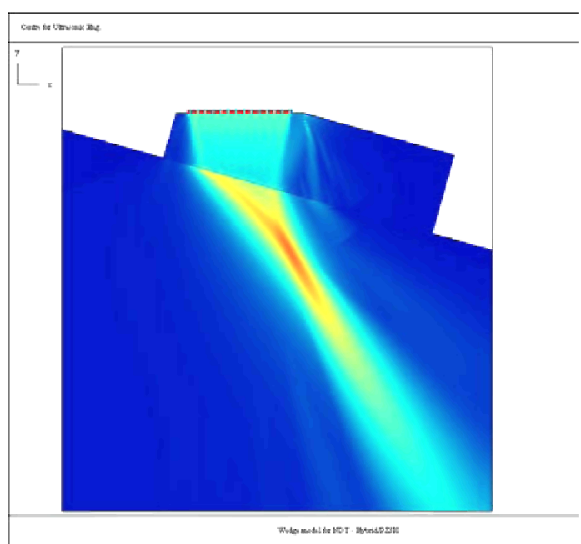

(b) PZT5H/hybrid configuration

FIGURE 6. Predicted peak pressure fields from two composite transducer configurations.

these two signals are clearly distinguishable in the PMN-PT/hybrid composite scenario, confirming to the improved bandwidth and imaging capabilities of this configuration. Furthermore, the focal law has been used to reconstruct the overall array pulse-echo response and these are presented in Figures 9(a) and 9(b) for the PZT5H/hard and PMNPT/hybrid composite cases respectively. From these Figures the enhanced imaging potential of the composite using the new active and passive materials is obvious as the two echoes, from the interface and defect, can be easily resolved.

\section{CONCLUSIONS}

This paper has demonstrated the imaging potential offered by the exploitation of new piezoelectric and passive materials used as the constituent materials in a piezoelectric composite structure. A finite element modeling approach has been used to investigate the NDE array performance of piezoelectric composite devices. Importantly, the combination

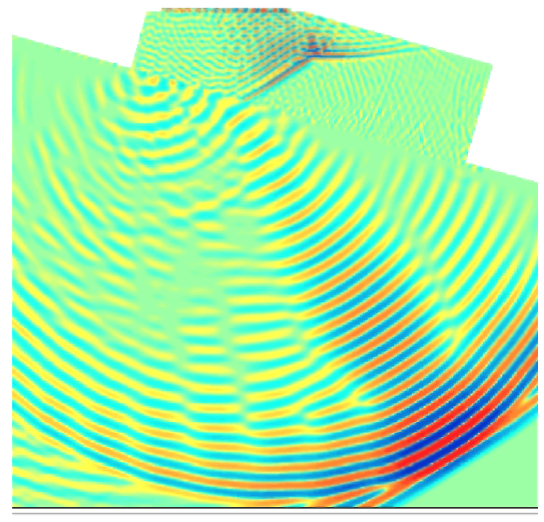

(a) PZT5H/hard configuration

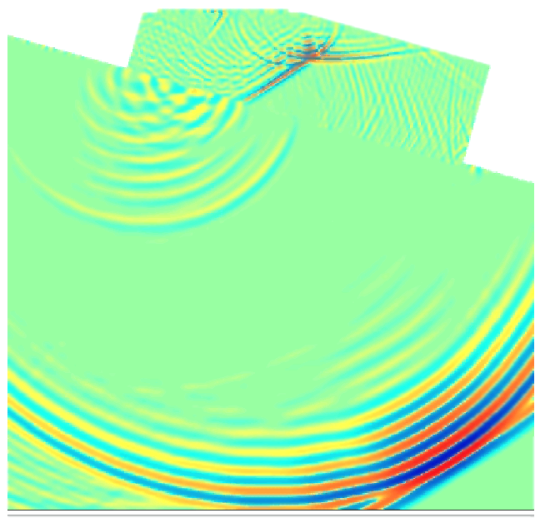

(b) PMN-PT/hybrid configuration

FIGURE 7. Predicted propagating pressure fields from two composite transducer configurations. 


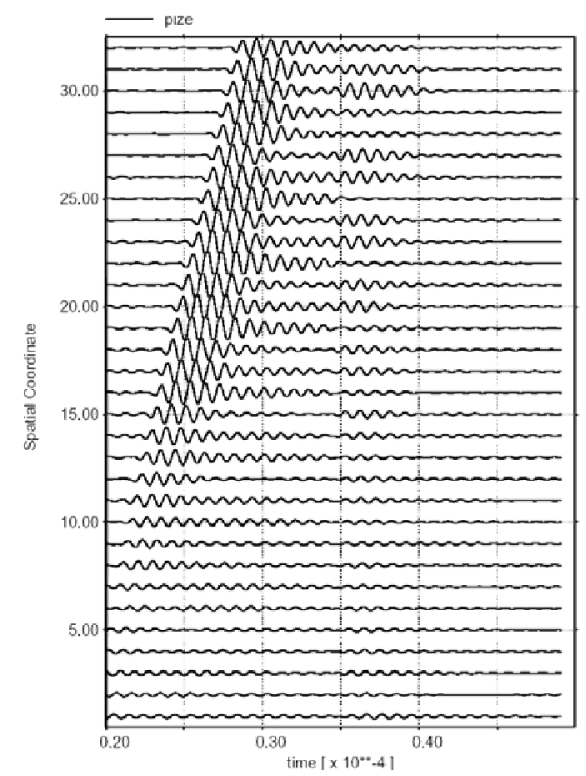

(a) PZT5H/hard configuration

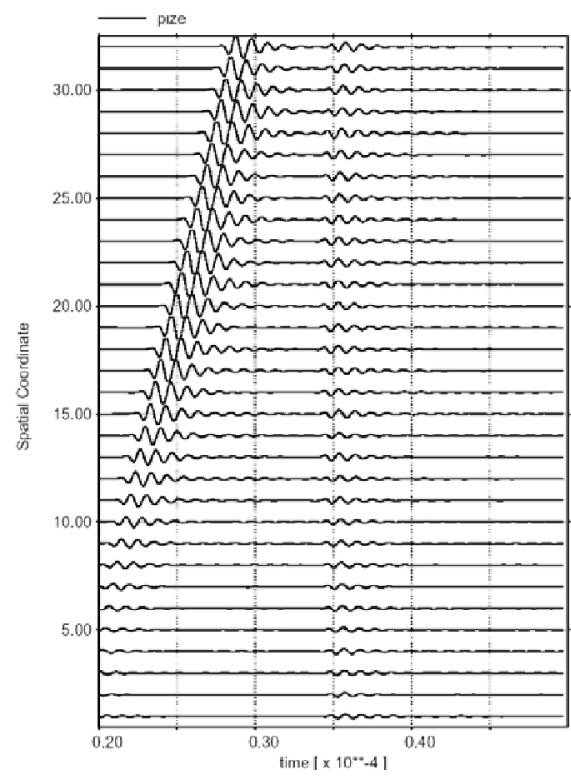

(b) PMN-PT/hybrid configuration

FIGURE 8. Individual Array Element Response from SDH defect.

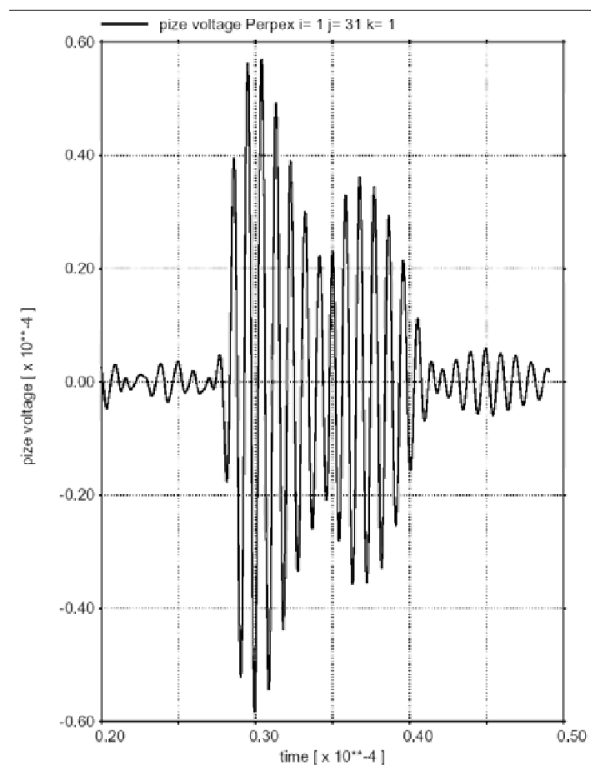

(a) PZT5H/hard configuration

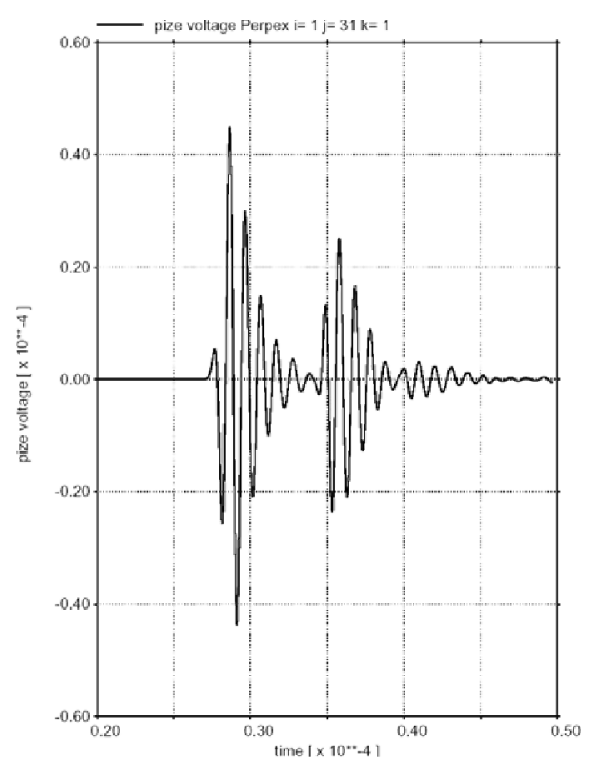

(b) PMN-PT/hybrid configuration

FIGURE 9. Predicted reconstructed defect response. 
of an active single crystal phase with a new polymer material developed at Strathelyde is predicted to realize a composite transducer array with enhanced bandwidth over conventional designs. Of course, the addition of backing and matching materials will improve the performance of conventional designs, but this will be at the expense of sensitivity. Consequently, the single crystal/hybrid composite array structure is extremely promising for application in the next generation of commercial ultrasonic array transducers.

\section{ACKNOWLEDGEMENTS}

The authors would like to acknowledge the contribution by Dr Agnes Parr, Mr Alexandre Troge, Professor Richard Pethrick and Professor Gordon Hayward for their work on EPSRC project (GR/S31235/01) developing the new polymer material and KTP programme (No 4450) on the application of single crystal materials in ultrasonic array transducers. Both of these research programmes contributed to this work which is funded through the UK Research Centre for Non-Destructive Evaluation (RCNDE).

\section{REFERENCES}

1. Harvey CJ, Pilcher JM, Eckersley RJ, Blomley MJK, Cosgrove DO, 'Advances in utrasound', Clinical Radiology 57 (3): 157-177 MAR 2002.

2. Benjamin $\mathrm{KC}$, 'Recent advances in 1-3 piezoelectric polymer composite transducer technology for AUV/UUV acoustic Imaging applications', Journal Of Electroceramics 8 (2): 145-154 AUG 2002.

3. Moles M, Dube N, Labbe S, Ginzel E, 'Review of ultrasonic phased arrays for pressure vessel and pipeline weld inspections', Journal Of Pressure Vessel Technology-Transactions Of The ASME 127 (3): 351-356 AUG 2005.

4. Gururaja, T.R., Schulze, W.A., Cross, L.E., Newnham, R.E., Auld, B.A., and Wang, Y., 'Piezoelectric Composite Materials for Ultrasonic Transducer Materials Part 1: Resonant Modes of Vibration in PZT Rod-Polymer Composites', IEEE Transactions on Sonics and Ultrasonics, Vol. 32, pp. 481-498, (1985).

5. Hossack, J.A. and Hayward, G., 'Finite-Element Analysis of 1-3 Composite Transducers', IEEE Transactions on Ultrason Ferroelect and Freq Contr, Vol. 38, pp. 618-629, (1991).

6. Hayward, G. and Bennett, J., 'Assessing the influence of pillar aspect ratio on the behaviour of 1-3 connectivity composite transducers', IEEE Trans UFFC, 43, No 1, Jan 1996, pp 98-108.

7. A.C.S. Parr, R.L. O'Leary and G. Hayward, 'Improving the Thermal Stability of 1-3 Piezoelectric Composite Transducers', Special Issue on ultrasonic transducers for high temperature applications, IEEE Transactions on Ultrasonics, Ferroelectries and Frequency Control, Vol 52(4), pp 550-564, 2005.

8. T. Ritter et al, "Single Crystal PZN/PT-Polymer Composites for Ultrasound Transducer Applications," IEEE Trans. Ultrason., Ferroelect., Freq. Contr., vol. 47, pp. 792-799, 2000.

9. Robertson, D., Hayward, G., Gachagan, A. and Murray, V. 'Comparison of the performance of pmn-pt single-crystal and ceramic composite arrays for NDE applications' Insight, Vol. 48, no. 2, pp. 97-100, (2006).

10. O'Leary, R.L., A.C.S Parr, A. Troge, R.A. Pethrick and G. Hayward, 'Performance of periodic piezoelectric composite arrays incorporating a passive phase exhibiting anisotropic properties' IEEE Ultrasonics Symposium Proceedings, pp. 1073-1076, 2005.

11. Hayward, G. and Hyslop J., 'Determination of Lamb Wave Dispersion Data in Lossy Anisotropic Plates Using Time Domain Finite Element Analysis. Parts I \& II' IEEE Transactions on Ultrasonics Ferroelectrics and Frequency Control, Vol. 53, pp. 443-455, (2006).

12. PZFlex User Manual, Weidlinger Associates, Inc., Consulting Engineers, 399 West El Camino Real, Suite 200, Mountain View, CA 94040-2607. 